\title{
Formation of Fe X-Fe XIV coronal lines in the accretion shock of $\mathrm{T}$ Tauri stars
}

\author{
S. A. Lamzin ${ }^{1}$, H. C. Stempels ${ }^{2}$, and N. E. Piskunov ${ }^{2}$ \\ 1 Sternberg Astronomical Institute, Moscow V-234, 119899 Russia \\ e-mail: lamzin@sai.msu.ru \\ 2 Uppsala Astronomical Observatory, Box 515, 75120 Uppsala, Sweden \\ e-mail: stempels@astro.uu.se
}

Received 26 October 2000 / Accepted 24 January 2001

\begin{abstract}
Specific intensities of the strongest Fe X-Fe XIV coronal lines were calculated in the framework of our accretion shock model (Lamzin 1998). These lines are formed in a region immediately behind the front of the accretion shock, therefore, the gas velocity in the line formation region is close to $1 / 4$ of the infall velocity. It appears that iron coronal lines in the optical band (e.g. [Fe x] $6376 \AA$ and [Fe XIV] $5304 \AA$ ) are too weak to be observed in spectra of T Tauri stars, but the UV lines (e.g. [Fe XI] $1467 \AA$ and [Fe XII] $1349 \AA$ ) can possibly be detected. In agreement with our calculations we could not detect the [Fe x] $6376 \AA$ and [Fe XIV] $5304 \AA$ lines in low noise UVES spectra of RU Lup where the accretion luminosity is ten times larger than the bolometric luminosity of the underlying star. At the same time we detected the [Fe XI] $1467 \AA$ line in a HST/GHRS spectrum of RY Tau which suggests that the accretion rate of the star in its quiescent state is $\approx 210^{-9} M_{\odot} /$ yr. As a byproduct of the study we found that for RY Tau the interstellar extinction coefficient $A_{V}$ is closer to $0.5^{\mathrm{m}}$ than to $1.0^{\mathrm{m}}$. For DF Tau, the observed upper limit for the flux of the [Fe XII] $1349 \AA$ line in HST/GHRS spectra is in agreement with an accretion rate of $210^{-9} M_{\odot} / \mathrm{yr}$ as found by Lamzin et al. (2000). As a critical test of our calculations we predict that the [Fe XI] $1467 \AA$ line in the spectrum of RU Lupi should be relatively strong: we expect the flux to be near $10^{-15} \mathrm{erg} / \mathrm{s} / \mathrm{cm}^{2}$.
\end{abstract}

Key words. stars: pre-main sequence - stars: individual: RU Lup, RY Tau, DF Tau - physical processes: accretion discs - physical processes: line formation - physical processes: shock waves

\section{Introduction}

Bisnovatyi-Kogan \& Lamzin (1977) have shown that if the continuum and the line emission observed in the spectra of classical T Tauri stars (CTTS) originate in chromospheric and coronal regions similar to solar ones, then the X-ray luminosity of young stars should be comparable to their bolometric luminosities. To detect hot coronal regions the authors proposed to search for coronal lines (CLs) in optical spectra of CTTS - that is for forbidden lines that correspond to transitions between fine structure levels of the ground configuration of highly ionized atoms in plasma with temperatures $T \gtrsim 10^{6} \mathrm{~K}$. Subsequent observations set very strict upper limits (down to $20 \mathrm{~m} \AA$ in some cases) on the intensities of the [Fe XIV] $5303 \AA$ and [Fe X] 6376 A CLs in spectra of CTTS. (Gahm et al. 1981; Gahm \& Krautter 1982; Lago et al. 1985), indicating that the nature of the emission spectra of young stars cannot be explained in terms of a simple analogy with solar activity.

Send offprint requests to: N. Piskunov, e-mail: piskunov@astro.uu.se
According to the modern scheme the emission spectrum of CTTS is the result of disk accretion onto magnetized low mass young stars. In the frame of this paradigm, the main portion of the line and continuum emission originates in the accretion shock that occurs when infalling matter collides with the stellar surface. Presumably, the infall velocity of the gas in CTTS is $V_{0} \simeq 300 \pm 100 \mathrm{~km} \mathrm{~s}^{-1}$, so the gas temperature just behind the shock front should be $\sim 1-310^{6} \mathrm{~K}$ (Königl 1991). Therefore, the accretion model does not only predict the regions with coronal temperatures, but does in some cases suggest that the accretion luminosity can be comparable or even exceeding the bolometric luminosity of the underlying young star. One of the main remaining questions is whether this accretion model is in agreement with the unsuccessful attempts to detect the CLs in spectra of CTTS.

The scope of this paper is a comparison between theoretically predicted intensities of iron CLs and spectral observations in the optical and UV spectral bands. The theoretical part of the paper is based on results of numerical calculations described by Lamzin (1998). All the 
details of the accretion shock structure discussed below are taken from this paper.

\section{Theoretical intensities of Fe X-XIV coronal lines}

The analysis of the energy balance in CTTS post-shock regions shows that the electron temperature does not exceed $310^{6} \mathrm{~K}$. Therefore, iron ions with a charge larger than +16 are practically absent in the accretion shock and we will concentrate on CLs of Fe X-Fe XIV ions (The ${ }^{1} \mathrm{~S}$ and ${ }^{2} \mathrm{~S}$ ground terms of the Fe XV and Fe XVI ions respectively have no fine splitting and therefore we expect no resonant coronal lines). The list of lines originating from transitions between levels of the ground configuration $3 \mathrm{~s}^{2} 3 \mathrm{p}^{n}$ of these ions is presented in Table 1 . The wavelength $\lambda_{i j}$ and transition probabilities $A_{i j}$ of the lines were adopted from the CHIANTI database (Landi et al. 1999). Only lines with $A_{i j}>1.0 \mathrm{~s}^{-1}$, i.e. with a sufficiently large critical density of electrons, were included in the table.

Table 1. Fe X-Fe XIV coronal lines

\begin{tabular}{llrrr}
\hline Ion & Config. & Transition & $\begin{array}{r}\lambda \\
{[\AA]}\end{array}$ & $\begin{array}{r}A_{i j} \\
{\left[\mathrm{~s}^{-1}\right]}\end{array}$ \\
\hline Fe X & $3 \mathrm{p}^{5}$ & ${ }^{2} \mathrm{P}_{3 / 2}-{ }^{2} \mathrm{P}_{1 / 2}$ & 6376.291 & 69.38 \\
Fe XI & $3 \mathrm{p}^{4}$ & ${ }^{3} \mathrm{P}_{2}-{ }^{3} \mathrm{P}_{1}$ & 7893.968 & 43.83 \\
& & ${ }^{3} \mathrm{P}_{1}-{ }^{1} \mathrm{D}_{2}$ & 3987.925 & 8.686 \\
& & ${ }^{3} \mathrm{P}_{2}-{ }^{1} \mathrm{D}_{2}$ & 2649.456 & 85.33 \\
Fe XII & $3 \mathrm{p}^{3}$ & ${ }^{3} \mathrm{P}_{1}-{ }^{1} \mathrm{~S}_{0}$ & 1467.420 & 974.8 \\
& & ${ }^{4} \mathrm{~S}_{3 / 2}-{ }^{2} \mathrm{D}_{3 / 2}$ & 2406.449 & 47.44 \\
& & ${ }^{4} \mathrm{~S}_{3 / 2}-{ }^{2} \mathrm{D}_{5 / 2} \mathrm{P}_{1 / 2}$ & 2169.762 & 1.887 \\
& & ${ }^{2} \mathrm{D}_{3 / 2}-{ }^{2} \mathrm{P}_{1 / 2}$ & 3071.913 & 70.46 \\
& & ${ }^{4} \mathrm{~S}_{3 / 2}-{ }^{2} \mathrm{P}_{3 / 2}$ & 1242.005 & 326.0 \\
Fe XIII & $3 \mathrm{p}^{2}$ & ${ }^{2} \mathrm{D}_{3 / 2}-{ }^{2} \mathrm{P}_{3 / 2}$ & 2566.735 & 195.4 \\
& & ${ }^{3} \mathrm{P}_{0}-{ }^{3} \mathrm{P}_{1}$ & 10746.217 & 14.01 \\
& & ${ }^{3} \mathrm{P}_{1}-{ }^{3} \mathrm{P}_{2}$ & 10797.737 & 9.867 \\
& & ${ }^{3} \mathrm{P}_{1}-{ }^{1} \mathrm{D}_{2}$ & 2579.820 & 62.00 \\
& & ${ }^{3} \mathrm{P}_{1}-{ }^{1} \mathrm{~S}_{0}$ & 1216.509 & 986.0 \\
& & ${ }^{3} \mathrm{P}_{1}-{ }^{1} \mathrm{D}_{2}$ & 2579.820 & 62.00 \\
Fe XIV & $3 \mathrm{p}$ & ${ }^{3} \mathrm{P}_{2}-{ }^{1} \mathrm{D}_{2}$ & 3389.693 & 75.57 \\
& & ${ }^{2} \mathrm{P}_{1 / 2}-{ }^{2} \mathrm{P}_{3 / 2}$ & 5304.196 & 60.22 \\
\hline
\end{tabular}

The width of the accretion post-shock zone is much smaller than the stellar radius, so one can treat the CLs emitting region as a plane-parallel gas slab. Let $\varepsilon\left(\mathrm{erg} / \mathrm{s} / \mathrm{cm}^{3}\right)$ be the volume emissivity of an optically thin CL, which belongs to a Fe ion with charge $Z$ corresponding to a transition from level $j$ to level $i$. Then

$\varepsilon=\frac{h c}{\lambda_{i j}} A_{i j} N_{j}=\frac{h c}{\lambda_{i j}} A_{i j} n_{j} n_{Z} \eta_{\mathrm{Fe}} N$,

where $N_{j}\left(\mathrm{~cm}^{-3}\right)$ is the particle number density of ions with excited level $j, n_{j}$ the relative population of the same level and $n_{Z}$ the relative abundance of the ion in question. We adopt the solar value of $3.710^{-5}$ for the relative iron abundance $\eta_{\mathrm{Fe}}$. Finally, $N\left(\mathrm{~cm}^{-3}\right)$ is the total particle number density.

The specific intensity $I_{\mathrm{n}}\left(\mathrm{erg} \mathrm{s}^{-1} \mathrm{~cm}^{-2} \mathrm{ster}^{-1}\right)$ of the line in the direction perpendicular to the slab surface (i.e. the $z$-axis of the slab) can be expressed as

$I_{\mathrm{n}}=\int \frac{\varepsilon}{4 \pi} \mathrm{d} z$,

and the observed flux as

$F=\frac{I_{\mathrm{n}} S_{\mathrm{ac}}}{d^{2}} \exp \left(-0.92 A_{\lambda}\right)$

(Gomez de Castro \& Lamzin 1999), where $S_{\mathrm{ac}}$ is the surface area of the accretion zone observed from the Earth; $d$ the distance to the star, and $A_{\lambda}$ the interstellar absorption coefficient at the wavelength of the CL in question.

The distribution of $T, N_{\mathrm{e}}$ and $n_{Z}$ along the flow can be obtained from numerical calculations of the accretion shock structure. We calculated $I_{\mathrm{n}}$ values for CLs from Table 1 as a function of the velocity $V_{0}$ of the infalling gas. We did this for two typical values of the gas particle density "at infinity" $N_{0}$, bearing in mind that the density of the infalling gas in CTTS is in the range $10.5 \leq \log N_{0} \leq$ 12.5 (see Lamzin 1995; Gomez de Castro \& Lamzin 1999). We used a five level atom model to calculate the relative level populations $n_{j}$. The necessary atomic data was taken from the CHIANTI database. Results of these calculations are shown in Fig. 1: curves for $\log N_{0}=11.0$ are shown with solid lines and curves for $\log N_{0}=12.0$ with dashed lines. The intensities of CLs do not seem to be too large: more than an order of magnitude less than the expected intensity of the Si III] $1892 \AA$ line (Gomez de Castro \& Lamzin 1999). Therefore only lines with $\lg I_{\mathrm{n}}^{\max } \geq 3$ are included in Fig. 1. Curves for the [Fe XIII] $1216.5 \AA$ and [Fe XII] $1242.0 \AA$ lines were not included, because, even though these lines have intensities in excess of the mentioned limit, these cannot be observed due to blending with much stronger lines (Ly $\alpha$ and N v $1242.8 \AA$ ).

Because a higher velocity of the infalling gas leads to a larger electron temperature $T_{\max }$ behind the shock front $\left(T_{\max } \propto V_{0}^{2}\right)$ and to a larger column density of highly ionized atoms, the $I_{\mathrm{n}}$ values increase monotonically with $V_{0}$ for all CLs. The only notable exception is the [Fe x] $6376.29 \AA$ line: its $I_{\mathrm{n}}$ value reaches a maximum at $V_{0} \simeq 350 \mathrm{~km} \mathrm{~s}^{-1}$, because the column density of Fe $\mathrm{X}$ ions decreases at higher infall velocities.

\subsection{Estimating the accretion rate}

The structure of the CTTS post-shock region depends little on densities in the range of $10.5 \leq \log N_{0} \leq 12.5$ (typical range assumed for infalling gas). More precisely, the value of $\int n_{Z} N \mathrm{~d} z$ is almost independent of $N_{0}$. This means that $I_{\mathrm{n}}$ can only depend on $N_{0}$ through the level population term $n_{j}$ in Eqs. (1) and (2). For the CLs of Fe XII and Fe XIV shown in Fig. 1 the relative population of the upper levels is proportional to their statistical 


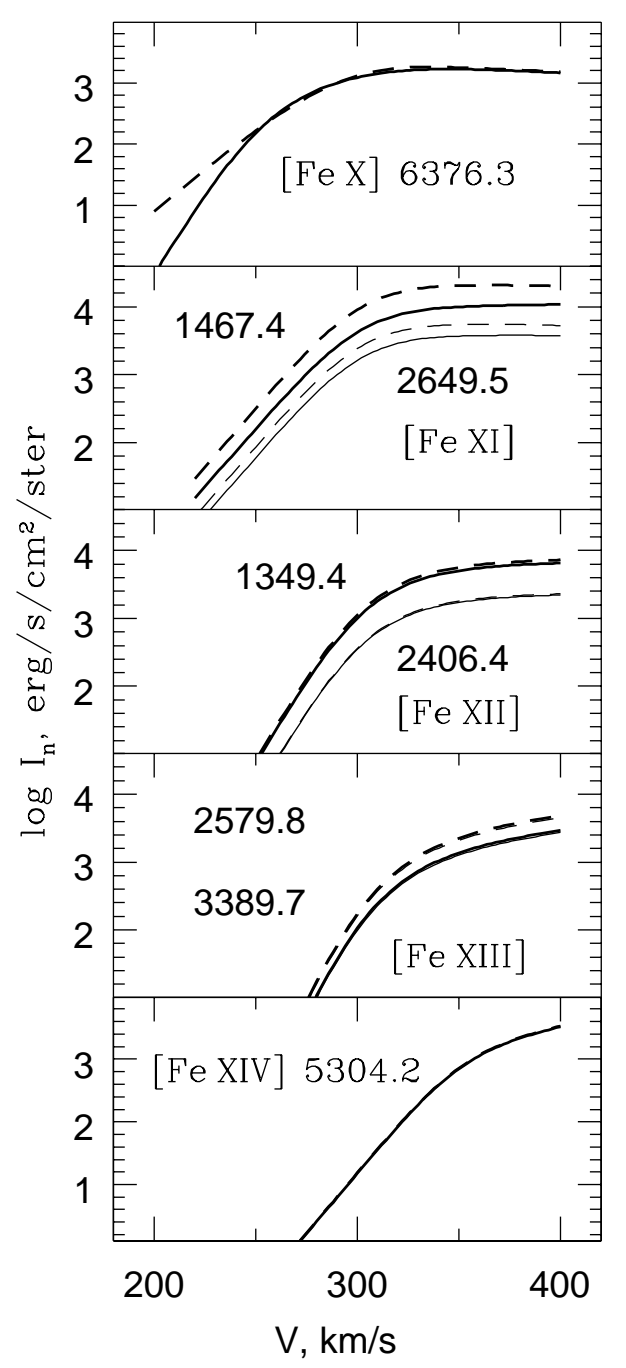

Fig. 1. Calculated specific intensities of the coronal lines of iron for $\log N_{0}=11.0$ (solid lines) and $\log N_{0}=12.0$ (dashed lines). In the case of Fe XI and Fe XII ions thin lines correspond to $2649.5 \AA$ and $2406.4 \AA \mathrm{CLs}$, while thick lines correspond to 1467.4 $\AA$ and 1349.4 $\AA$ CLs. The lines corresponding to [Fe XIII] $2579.8 \AA$ and [Fe XIII] $3389.7 \AA$ CLs practically coincide on the scale of the figure

weights, i.e. independent of $N_{\mathrm{e}}$, because the electron density in the line formation region of these lines is $N_{\mathrm{e}} \simeq 4 N_{0}$, which is significantly larger than the critical value $N_{\mathrm{e}}^{\mathrm{cr}}$ attributed to collisional quenching. From this it follows that these line intensities appears to be almost independent of $N_{0}$. In the case of other CLs shown in Fig. 1, $N_{\mathrm{e}}$ is of the order of $N_{\mathrm{e}}^{\mathrm{cr}}$, and thus $I_{\mathrm{n}}$ depends significantly on $N_{0}$, but not as strong as in the case of optically thin lines of dipole allowed transitions, for which both $n_{j}$ and $I_{\mathrm{n}}$ should be proportional to $N_{0}$.

From Eq. (3) one can derive the surface area $S_{\mathrm{ac}}$ of the part of the accretion zone that is visible from the Earth by measuring the observed flux in a given coronal line. Assuming that due to projection only half of the total surface area of the accretion zone is visible, it is possible to estimate the accretion rate as follows: $\dot{M}_{\mathrm{ac}}=2 S_{\mathrm{ac}} V_{0} N_{0} m_{\mathrm{p}} \mu$, where $m_{\mathrm{p}}$ is the proton mass and $\mu \simeq 1.3$ the mean molec- ular weight of accreted gas. Introducing the parameter $\xi_{\lambda}=\exp \left(-0.92 A_{\lambda}\right)$ and expressing $V_{0}$ in $\mathrm{kms}^{-1}, N_{0}$ in $\mathrm{cm}^{-3}, d$ in pc, $F$ in $\mathrm{erg} / \mathrm{s} / \mathrm{cm}^{2}$, and $I_{\mathrm{n}}$ in $\mathrm{erg} / \mathrm{s} / \mathrm{cm}^{2} / \mathrm{ster}$, one can write finally:

$$
\begin{aligned}
\dot{M}_{\mathrm{ac}}\left(\frac{M_{\odot}}{\mathrm{yr}}\right)= & \frac{5.910^{-8}}{\xi_{\lambda}}\left(\frac{N_{0}}{310^{11}}\right)\left(\frac{V_{0}}{300}\right) \\
& \times\left(\frac{F}{10^{-15}}\right)\left(\frac{d}{100}\right)^{2}\left(\frac{10^{3}}{I_{\mathrm{n}}}\right) .
\end{aligned}
$$

\section{Comparison with observations}

\subsection{The optical band}

It is necessary to understand whether iron CLs can be seen at all in spectra of CTTS. From our calculations it follows that two of the strongest lines can be found in the optical band, while all other lines have $\lambda<3400 \AA$. At first glance it seems more attractive to search for CLs in the optical band with ground based telescopes, rather than in the UV band from space. However, the stellar contribution is maximal in the optical band, which may prevent detection of CLs. To illustrate this let us estimate the expected relative intensities of the [Fe X] $6376.3 \AA$ and [Fe XIV] $5304.2 \AA$ lines in CTTS spectra, assuming that their specific intensity $I_{\mathrm{n}} \sim 10^{3} \mathrm{erg} / \mathrm{s} / \mathrm{cm}^{2} / \mathrm{ster}$ (see Fig. 1).

Consider a star radiating as a blackbody $\left(I_{\lambda}(\theta)=\right.$ $\left.B_{\lambda}\right)$ with $T_{\text {eff }}=4000 \mathrm{~K}$ and assume that the accretion zone covers $10 \%$ of the stellar surface $S_{*}$. Then the observed monochromatic flux $F_{\lambda}$ from this star is equal to $\xi_{\lambda} B_{\lambda} S_{*} / 4 d^{2}$. At $\lambda=6000 \AA B_{\lambda}(4000 \mathrm{~K}) \simeq$ $410^{5} \mathrm{erg} / \mathrm{s} / \mathrm{cm}^{2} / \mathrm{ster} / \AA$. Coronal lines form in the region just behind the shock front, where the infalling gas moves with a velocity $\simeq V_{0} / 4$, thus one can expect that the FWHM of CLs should be of the same order of magnitude: around $80 \mathrm{kms}^{-1}$ which corresponds to $1.6 \AA$ at $\lambda=6000 \AA$. Writing $F_{\max }=F / F W H M$, one finds from these data and Eq. (3) that the expected maximum intensity $F_{\max }$ of these CLs is almost 1000 times less than intensity of the underlying stellar continuum, which means that the equivalent width of the lines $W=F / F_{\lambda}$ translates to $1 \mathrm{m \AA}$.

In addition, continuum emission originating in the accretion shock and in the innermost part of an accretion disc decreases the equivalent width of the CLs, because the larger the expected intensity is, the larger is the intensity of the veiling continuum. For example, in the case of the extremely active CTTS RU Lup, the accretion luminosity is almost 10 times larger than the bolometric luminosity of the underlying photosphere. The corresponding accretion rate is $\dot{M} \simeq 310^{-7} M_{\odot} / \mathrm{yr}$, where we used $A_{V}=0.3^{\mathrm{m}}$ and $d=200$ pc (Lamzin et al. 1996; but see also Bertout et al. 1999). Lamzin (2000a) also derived $V_{0}$ to be $300 \mathrm{~km} \mathrm{~s}^{-1}$ and $N_{0} \simeq 310^{12} \mathrm{~cm}^{-3}$, so from Eq. (4) and Fig. 1 one can estimate the flux of the [Fe X] $6376.3 \AA$ or [Fe XIV] $5304.2 \AA$ CLs to be around $210^{-16} \mathrm{erg} / \mathrm{s} / \mathrm{cm}^{2} / \AA$. 

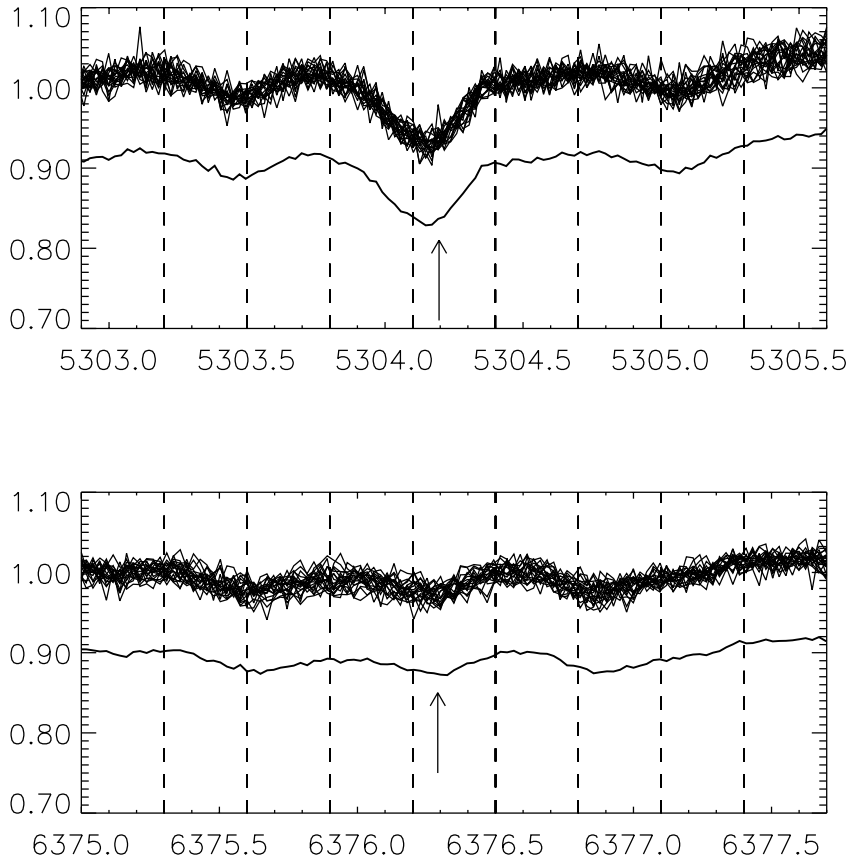

Fig. 2. 20 spectra of RU Lupi taken with VLT/UVES over two consecutive nights. The thin line is the average spectrum, displaced by -0.10 . The top panel shows the region around the [Fe XIV] $5304.2 \AA$ line, the bottom panel the [Fe x] $6376.3 \AA$ line. The wavelength positions of the lines are indicated by arrows

As an illustration we compare this calculation with high-resolution spectra $(R \approx 60000)$ of RU Lupi taken with the UVES spectrograph at the VLT on 16 and 17 April 2000. These spectra are shown in Fig. 2. The $S / N$ ratio per resolution element of each of the spectra is better than 200. As can be seen from this figure, no emission features are visible. This is in agreement with the calculation in the previous paragraph. Indeed, assuming that the star is in its quiescent state $\left(V=11^{\mathrm{m}}\right)$, the $S / N$ of the average spectrum in Fig. 2 corresponds to a detection limit $\sigma$ of $210^{-16} \mathrm{erg} / \mathrm{s} / \mathrm{cm}^{2} / \AA$ in a single resolution element. Therefore, we do not expect to be able to see the [Fe X] $6376.3 \AA$ or [Fe XIV] $5304.2 \AA \mathrm{CLs}$ in the observations, even if the CL would be as narrow as a single resolution element $(0.1 \AA)$.

\subsection{The UV band}

The observed intensity of the continuum in CTTS decreases rapidly shortward of $3000 \AA$; therefore the contrast and equivalent widths of CLs should be much larger in the UV than in optical band. On the other hand, interstellar absorption is significantly larger in this spectral band, so generally speaking it is not possible to predict a priori if one indeed can observe CLs in UV spectra of CTTS. Consider for example the low resolution $(R=2000) \mathrm{HST} / \mathrm{GHRS} z 2 \mathrm{dl0207t}$ spectrum of RY Tau in the vicinity of [Fe XI] $1467.4 \AA \mathrm{CL}$, which is shown in Fig. 3. This spectrum is part of Fig. 3 in Lamzin (2000b).

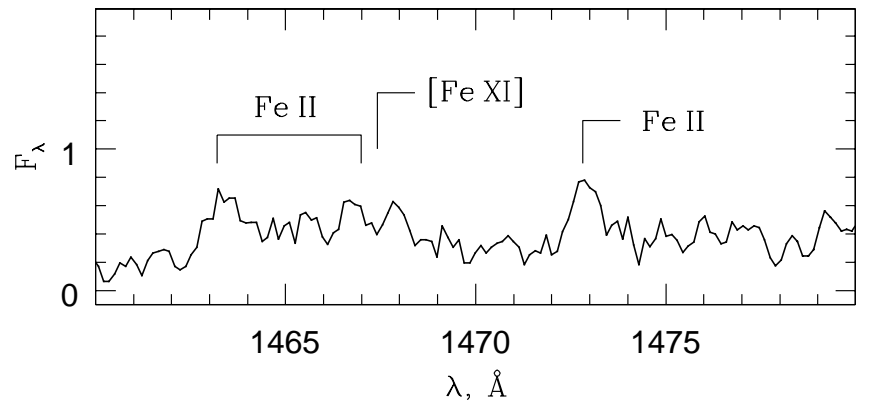

Fig. 3. A part of HST/GHRS z2dl0207t spectrum of RY Tau in the vicinity of [Fe XI] $1467.4 \AA$ coronal line. $Y$-axis is labelled in units $10^{-15} \mathrm{erg} \mathrm{s}^{-2} \mathrm{~cm}^{-2} \AA^{-1}$. See text for details

We could identify two lines of the Fe II $a^{4} \mathrm{G}-w^{4} \mathrm{G}^{\circ}$ multiplet ( $\lambda \lambda 1463.20$ and $1466.99 \AA$ ) and one of the Fe II $a^{4} \mathrm{D}_{3 / 2}-x^{2} \mathrm{P}_{1 / 2}^{\mathrm{o}}$ multiplet $(\lambda 1472.82 \AA)$ in the spectrum. There is an emission feature redward $\left(\Delta V \simeq+80 \mathrm{~km} \mathrm{~s}^{-1}\right)$ of the expected position of the [Fe XI] $1467.4 \AA$ line.

If the redshift is the result of gas motion with $V \simeq V_{0} / 4$ in the line formation region, this is possibly the CL; we could not find any other reasonable identification of this feature. The feature is unresolved and the integrated line flux is around $10^{-16} \mathrm{erg} / \mathrm{s} / \mathrm{cm}^{2}$.

The [Fe XII] $1349.4 \AA$ line is also within the wavelength range of the $z 2 \mathrm{dl} 0207 \mathrm{t}$ spectrogram, but we could not detect it. This is in agreement with our theoretical prediction: from Fig. 1 one can deduce that the specific intensity of this line should be at least two times smaller than that of the [Fe XI] $1467.4 \AA$ line, implying that the expected flux of the [Fe XII] $1349.4 \AA$ line would be below the noise level of the observed spectrum at $\lambda=1349 \AA$.

According to Lamzin (2000b) the density of the infalling gas in RY Tau is $\sim 1-210^{11} \mathrm{~cm}^{-3}$ and $V_{0} \simeq$ $350 \mathrm{~km} \mathrm{~s}^{-1}$. From Fig. 1 it follows that the specific intensity of the [Fe XI] $1467.4 \AA$ line is $\simeq 10^{4} \mathrm{erg} / \mathrm{s} / \mathrm{cm}^{2} / \mathrm{ster}$. To derive the accretion rate from Eq. (4) we need to know the interstellar absorption coefficient $A_{V}$, of which estimates range from $0.55^{\mathrm{m}}$ (Hartigan et al. 1995) to $1.3^{\mathrm{m}}$ (Petrov et al. 1999). Within this uncertainty, the coefficient $\xi_{\lambda}$ in Eq. (4) at $\lambda=1467 \AA$ varies between 0.26 and 0.041 , adopting the normal interstellar extinction law (Seaton 1979). To decrease the uncertainty in $A_{V}$ we took a UV spectrum of RY Tau observed with the IUE satellite from the INES database. In Fig. 4, we show the observed spectrum LWP17186LL in the top panel, and dereddened spectra with $A_{V}=0.5^{\mathrm{m}}$ and $A_{V}=1.0^{\mathrm{m}}$ in the middle and bottom panels respectively. Although the $S / N$-ratio of the original spectrum below $2400 \AA$ is not very good, one can see that dereddening with $A_{V}=1.0^{\mathrm{m}}$ produces an artificial flattening of the RY Tau spectrum below $2500 \AA$, which is due to a local maximum of the interstellar absorption coefficient around $2150 \AA$. We conclude therefore that the value $A_{V}=0.55^{\mathrm{m}}$ of Hartigan et al. seems more realistic. 


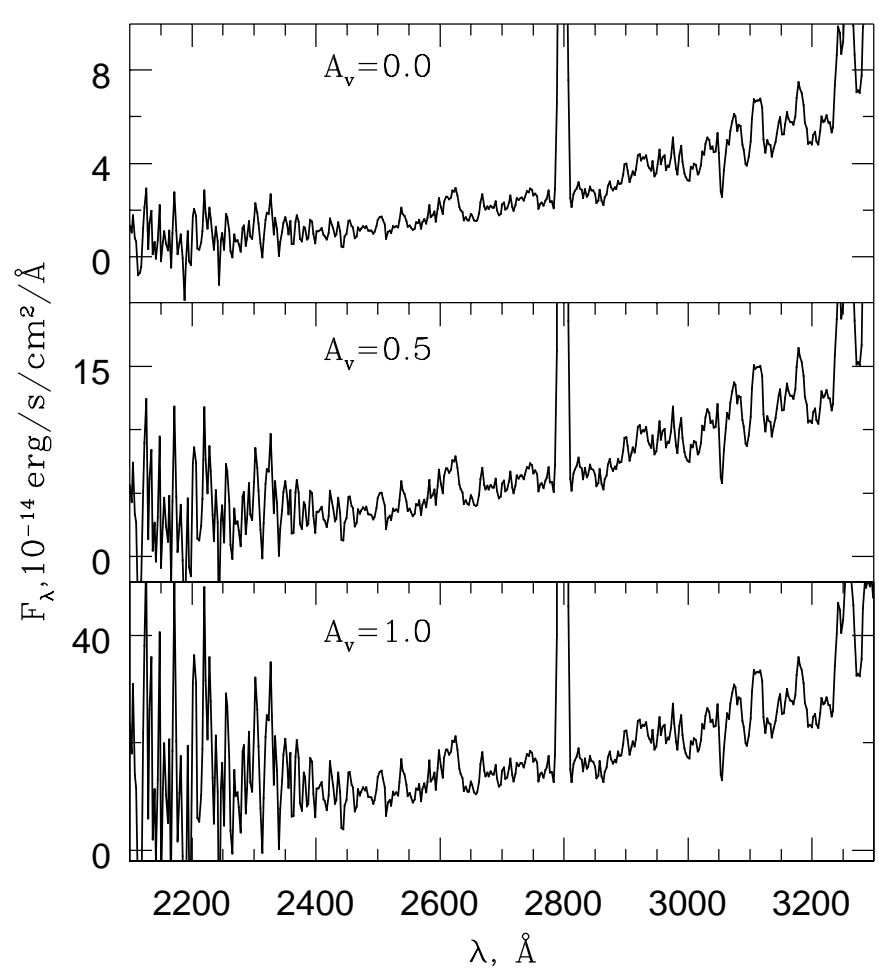

Fig. 4. Top panel: IUE LWP17186LL spectrum of RY Tau, middle and bottom panels: the same spectrum, but dereddened with $A_{V}=0.5^{\mathrm{m}}$ and $A_{V}=1.0^{\mathrm{m}}$ respectively

One can now find from Eq. (4) and $d=140 \mathrm{pc}$ that the accretion rate of RY Tau is around $210^{-9} M_{\odot} / \mathrm{yr}$. Using the value of $R=2.4 R_{\odot}$. for the stellar radius (Hartigan et al. 1995) this means that according to Eq. (2) the accretion zone occupies $\sim 10-20 \%$ of the stellar surface. Thus the parameters of the accretion process, derived from the observed intensity of this line (presumably [Fe XI] 1467.2 $\AA$ ) look reasonable. Note however that HST spectrum z2dl0207t was obtained on November 13, 1994, i.e. two years before the October 1996 flare, during which RY Tau increased its brightness up to $V=9.6^{\mathrm{m}}$ (Petrov et al. 1999). This means that the accretion rate we derived refers to the pre-flare period, when $V$ was $\simeq 11^{\mathrm{m}}$ - see the W. Herbst database (Herbst et al. 1994).

Another example: Lamzin et al. (2000) found the following parameters of DF Tau from analysis of UV spectra observed with IUE and HST: $d \simeq 70$ pc; $A_{V} \simeq 0.5^{\mathrm{m}} ; V_{0} \simeq 250 \mathrm{~km} \mathrm{~s}^{-1} ; \lg N_{0} \simeq 11$ and $\dot{M} \simeq 310^{-9} M_{\odot} /$ yr. Substituting these data and $I_{\mathrm{n}} \simeq 610^{3} \mathrm{erg} / \mathrm{s} / \mathrm{cm}^{2} / \mathrm{ster}$ (from Fig. 1) in Eq. (4) one finds that the expected flux of the [Fe XII] $1349 \AA$ line should be around $510^{-16} \mathrm{erg} / \mathrm{s} / \mathrm{cm}^{2}$, which is in agreement with the upper limit of $10^{-15} \mathrm{erg} / \mathrm{s} / \mathrm{cm}^{2}$ found by Lamzin et al. (2000) from HST/GHRS spectrum $\mathrm{z} 18 \mathrm{e} 0209 \mathrm{~m}$. The accretion rate values found by Gullbring et al. (1998) $\left(\sim 10^{-7} M_{\odot} / \mathrm{yr}\right)$ and Hartigan et al. (1995) ( $\left.>10^{-6} M_{\odot} / \mathrm{yr}\right)$ are inconsistent with our observed upper limit of the CL flux. We attribute this disagreement to the limitations of the theoretical models used in these papers (an isothermal gas slab in one case and a boundary layer in the other). In addition, measurements of accretion rates through CLs reflect the situation at a given moment. In order to compare our accretion rates with outflow rates one should monitor CLs over a longer period, because outflow rates are intrinsically time-integrated values.

Note finally that in the case of RU Lup one can expect that the [Fe XI] $1467 \AA$ line should be relatively strong: we expect its flux to be around $10^{-15} \mathrm{erg} / \mathrm{s} / \mathrm{cm}^{2}$. Unfortunately, we have no possibility to test this prediction at this moment.

\section{Conclusions}

From our calculations it follows that the intensities of the Fe X and Fe XIV coronal lines in spectra of CTTS should be relatively low. Apparently one cannot expect to detect coronal lines in optical spectra of these objects due to a low contrast between CLs and the relatively strong underlying continuum. In particular we could not detect the [Fe x] $6376 \AA$ and [Fe XIV] $5304 \AA$ lines in the case of RU Lupi, for which the accretion luminosity is almost ten times larger than the bolometric luminosity of the star itself, in spite of the fact that the $S / N$-ratio of our UVES spectra was better than 200 .

Some UV iron coronal lines (e.g. [Fe XI] $1467 \AA$ and [Fe XII] $1349 \AA$ ) have larger specific intensities than the strongest optical CLs, and we demonstrated that these lines can in principle be detected in CTTS spectra. Along with other relevant information, observed fluxes of iron UV coronal lines can be used to derive important parameters of the accretion process, such as the accretion rate.

The accuracy of the estimated accretion rates depends strongly on the uncertainties in the distance and the interstellar extinction. Long-term monitoring of CLs is needed to establish the balance between the mean accretion rate and the outflow rate.

Acknowledgements. Archival spectra extracted from the INES and HST databases were used in the paper. The P. van Hoof database (http://www.pa.uky.edu/ peter/atomic) was used for line identification. We thank T.P. Ray, the referee, for useful remarks that helped to improve the paper.

\section{References}

Bisnovatyi-Kogan, G. S., \& Lamzin, S. A. 1977, Sov. Astron., 21,720

Bertout, C., Robichon, N., \& Arenou, F. 1999, A\&A, 352, 574 Gomez de Castro, A. I., \& Lamzin, S. A. 1999, MNRAS, 304, $41 p$

Gahm, G .F., Lago, M. T. V. T., \& Penston, M. V. 1981, MNRAS, 195, 59p

Gahm, G. F., \& Krautter, J. 1982, A\&A, 106, 25

Gullbring, E., Hartmann, L., Briceno, C., \& Calvet, N. 1998, ApJ, 492, 323 
Hartigan, P., Edwards, S., \& Ghandour, L. 1995, ApJ, 452, 736

Herbst, W., Herbst, D. K., Grossman, E. J., \& Weinstein, D. 1994, AJ, 108, 1906.

Königl, A. 1991, ApJ, 370, L39.

Lago, M. T. V. T., Penston, M. V., \& Johnstone, R. M. 1985, MNRAS, 212, 151

Lamzin, S. A., Bisnovatyi-Kogan, G. S., Errico, L., et al. 1996, A\&A, 306, 877

Lamzin, S. A. 1998, Astron. Reports, 42, 322
Lamzin, S. A. 2000a, Astron. Lett., 26, 225

Lamzin, S. A. 2000b, Astron. Lett., 26, 589

Lamzin, S. A., Vittone, A. A., \& Errico, L. 2000, Astron. Lett., in press

Landi, E., Landini, M., Dere, K. P., Young, P. R., \& Mason, H. P 1999, A\&AS, 135, 339

Petrov, P. P, Zajtseva, G. V., Efimov, Yu. S., et al. 1999, A\&A, 341,553

Seaton, M. J. 1979, MNRAS, 187, 73P 